\title{
The Mosaic of Primary Care Nurses in Rural and Remote Canada: Results from a National Survey
}

\author{
Mosaïque de la main-d'œuvre infirmière en soins \\ primaires dans les régions canadiennes rurales et \\ éloignées : résultats d'une enquête nationale
}

RUTH MARTIN-MISENER, PHD, NP, FAAN

Professor and Director, School of Nursing, and Assistant

Dean, Faculty of Health

Dalhousie University

Halifax, NS

MARTHA L.P. MACLEOD, PHD, RN

Professor and the Northern Health - UNBC Knowledge

Mobilization Research Chair, School of Nursing

University of Northern British Columbia

Prince George, BC

ERIN C. WILSON, PHD, NP(F)

Assistant Professor, School of Nursing

University of Northern British Columbia

Prince George, BC

JULIE G. KOSTENIUK, PhD

Professional Research Associate, Canadian Centre for

Health and Safety in Agriculture

University of Saskatchewan

Saskatoon, SK
KELLY L. PENZ, PHD, RN

Assistant Professor, College of Nursing

University of Saskatchewan, Regina Campus

Regina, SK

NORMA J. STEWART, PHD

Professor Emerita, College of Nursing

University of Saskatchewan

Saskatoon, SK

JANNA OLYNICK, MSC

Research Associate, School of Nursing

University of Northern British Columbia

Prince George, BC

CHANDIMA P. KARUNANAYAKE, PHD Professional Research Associate, Canadian Centre for Health and Safety in Agriculture

University of Saskatchewan

Saskatoon, SK 


\begin{abstract}
Background and objective: Nurses provide essential primary care (PC) in rural and remote Canada. We examined the practice context and responsibilities of this little-known understudied workforce.

Method: Data from Nursing Practice in Rural and Remote Canada II, a 2014 to 2015 panCanadian survey, were analyzed.

Results: Of 3,822 respondents, 192 identified that PC was their only practice focus (PC-Only), and for 111, it was one focus among others (PC-Plus). Proportionally more PC-Only than PC-Plus nurses had graduate education, were employed in larger communities and had experienced higher job resources and lower job demands. Proportionally fewer PC-Only than PC-Plus nurses followed protocols/decision support tools, dispensed medications and provided emergency services. Proportionally more PC-Only than PC-Plus nurses ordered advanced diagnostic tests/imaging, and fewer PC-Only than PC-Plus nurses performed and interpreted laboratory tests and diagnostic imaging on site.

Conclusion: Contributions of the rural and remote nursing workforce to $\mathrm{PC}$ are rendered invisible by contemporary characterizations of the PC workplace, limiting evaluation and improvement efforts.
\end{abstract}

\title{
Résumé
}

Contexte et objectif: Les infirmières fournissent des soins de santé primaires (SSP) essentiels dans les régions rurales et éloignées du Canada. Nous avons examiné le contexte et les responsabilités de la pratique de cette main-d'œuvre peu connue et mal étudiée.

Méthode: Nous avons analysé des données provenant d'une enquête pancanadienne sur la pratique infirmière dans les régions canadiennes rurales et éloignées, de 2014 à 2015 (Nursing Practice in Rural and Remote Canada II).

Résultats: Parmi 3822 répondants, 192 ont indiqué que les SSP formaient l'essentiel de leur pratique (SSP-seul) et 111 ont indiqué que ces soins formaient une partie de leur pratique (SSP-plus). Toute proportion gardée, plus d'infirmières SSP-seul que SSP-plus ont reçu leur diplôme, ont été employées dans des grandes communautés, ont bénéficié de plus de ressources au travail et ont connu moins d'exigences au travail. Toute proportion gardée, moins d'infirmières SSP-seul que SSP-plus ont utilisé les outils d'aide aux protocoles ou à la décision, ont délivré des médicaments et ont fourni des services d'urgence. Toute proportion gardée, plus d'infirmières SSP-seul que SSP-plus ont prescrit une imagerie ou un test diagnostic poussé et moins d'infirmières SSP-seul que SSP-plus ont effectué et interprété des tests en laboratoire et des imageries diagnostiques sur les lieux.

Conclusion: La contribution de la main-d'œuvre infirmière dans les régions canadiennes rurales et éloignées demeure invisible en raison des caractérisations contemporaines du lieu de travail des SSP, ce qui limite les efforts d'évaluation et d'amélioration. 


\section{Introduction}

Canada has experienced nearly two decades of incremental reforms in community-based primary healthcare (Hutchison 2013). Many of the changes have occurred in primary care (PC), which we understand is the first point of entry to and an ongoing point of contact with the overall healthcare system (Starfield 1992). Most Canadians receive care for the majority of their health concerns in PC, from birth to death, often with the same healthcare provider (Statistics Canada 2017).

Nurses now provide PC services in all provinces and territories (Martin-Misener et al. 2014). This is a dramatic change for nurses in southern regions of the country, but less so for nurses in rural and remote regions, where the vastness of Canada's land mass and low population density make PC health service delivery particularly challenging (Martin et al. 2018). Changes to the roles and responsibilities of physicians and nurses, sometimes called taskshifting, is a world wide strategy intended to improve accessibility to PC (Maier and Aiken 2016). Evidence from the US indicates that as PC physician recruitment for rural areas is declining, nurse practitioner (NP) recruitment is increasing (Barnes et al. 2018; Xue et al. 2019). A Canada-wide survey of nurses in rural and remote Canada found that twice as many registered nurses (RNs) working in PC versus RNs working in other care settings reported working beyond their scope of practice (MacLeod et al. 2019). Nurses have been the backbone of rural and remote PC for more than 50 years, yet very little is known about their deployment and practice.

The Canadian Institute for Health Information (CIHI) details the supply, distribution and practice characteristics of NPs, RNs, licensed practical nurses (LPNs) and registered psychiatric nurses (RPNs) in an annual regulated nurse report, the most recent of which is Regulated Nurses 2018 (CIHI 2019a, b), and in the Pan-Canadian Primary Health Care Indicator Update Report (CIHI 2012). The CIHI-regulated nurse report uses registration data collected by provincial/territorial nurse regulatory organizations to identify trends in supply, distribution, employment and demographic characteristics. Place of work is grouped into the following four categories: hospital (hospital, mental health centre and rehabilitation/convalescent centre); community health agency (nursing station [outpost or clinic], home care agency, community health centre and public health department/unit); nursing home/ long-term care facility; and other place of work (business/industry/occupational health office, private nursing agency/private duty, self-employed, physician's office/family practice unit, educational institution, nursing association/government and other). CIHI's indicator report defines workplace for a PC nurse as "a nursing station, community health centre, or a physician's office/family practice unit" (CIHI 2012, p. 109). Valuable as these definitions are, defining PC nurses using their self-reported practice location can be problematic in rural and remote settings that do not have a clear delineation of specialized places of work as seen in urban settings. Rural and remote healthcare contexts often require nurses to work in multiple practice areas, for example, home and acute care, PC and community care (NNPBC 
2018). Therefore, equating workplace with type of health services offered may be inappropriate, particularly in smaller communities (Smith and Vandall-Walker 2017).

Health workforce solutions are needed to address the health needs of rural and remote populations that have high proportions of older adults and some of the highest rates of all-cause mortality in the country (Tam 2017). In Canada's rapidly changing healthcare environment, not knowing which nurses are providing PC and where they are employed is a serious information gap in planning and providing accessible high-quality rural and remote health services (British Columbia Ministry of Health 2015). The purpose of this study was to examine the practice context and responsibilities of PC nurses in rural and remote Canada.

\section{Method}

\section{Design}

Data for this analysis are from a pan-Canadian cross-sectional survey - Nursing Practice in Rural and Remote Canada II. MacLeod et al. (2017) provide a detailed description of the methods used. The definition of rural encompasses areas beyond the commuting zones of communities with 10,000 or more residents (du Plessis et al. 2001). Remote was not specifically defined. We used the phrase "rural and remote" because findings of previous research have shown more similarities than differences in how nurses define rural and remote workplaces (Kulig et al. 2008). The comprehensive questionnaire (27 pages in English/ 31 pages in French) was created based on a previous work (MacLeod et al. 2004; Stewart et al. 2005) and an integrated view of workforce planning (Tomblin Murphy et al. 2009). The questionnaire was developed iteratively by a 16 -member research team with guidance from a 19-member advisory group composed of provincial and national nurse decision-makers. It was mailed in 2014 to 2015 to 10,072 regulated nurses practicing in rural and remote communities in each province and all regulated nurses in the territories.

\section{Participants}

A total of 3,822 out of the 9,622 eligible respondents completed the survey, resulting in a $40 \%$ response rate $(N=3,822)$. The questions that were used to identify the subsample of rural and remote PC nurses (staff nurses, NPs, clinical nurse specialists and managers) included those on primary place of employment and area of practice. Options for PC place of employment included those in CIHI's (2012) definition, such as nursing station, community health centre or physician's office/family practice unit, with additions of nursing stations/ outpost clinics/nurse clinics, family practice teams, NP-led clinics, multidisciplinary primary healthcare clinics. Only one response was permitted for this question. For area of current practice, respondents had the option of choosing all that applied from the following: acute care, primary care, community health, long-term care, home care, hospice/end-of-life/palliative care, mental health, and other. RPN respondents were excluded from the PC analysis owing to the small sample size $(N=29)$. 


\section{Measures}

Survey content areas used in this analysis were as follows: individual (nurse type, gender, age, highest nursing education credential, region of residence and primary position), work community (population size and distance from advanced referral centre), workplace (required to be on call, job resources, job demands and primary health care engagement) and practice responsibilities (e.g., assessment [nine items], laboratory tests [five items], diagnostic tests [four items], diagnostic imaging [five items], diagnosis and referral [five items], therapeutic management [five items], emergency care and transportation [four items]). For brevity, we report on the subset of responsibilities that most distinguish the differences.

The 40-item Primary Health Care Engagement (PHCE) scale was composed of $10 \mathrm{sub}$ scales, each with three to five items (Kosteniuk et al. 2017). Items were rated on a five-point scale $(1=$ strongly disagree to $5=$ strongly agree). The items in each subscale were summed and averaged to produce a subscale score indicating low agreement (1.0-3.0) or high agreement (>3.0). Higher scores represent perceptions of greater workplace engagement in PHC delivery. The 24-item Job Resources in Nursing (JRIN) scale and the 22-item Job Demands in Nursing (JDIN) scale were used to measure the overall job-related demands and resources across organizational, interpersonal and job position/task levels (Penz et al. 2018). The total summated scores ranged from 24 to 120 for the JRIN, interpreted as low (24-56), medium (57-88) or high (89-120) level of work-related resources. Similarly, the total summated scores on the JDIN ranged from 22 to 110, interpreted as low (22-51), medium (52-80) or high (81-110) level of work-related demands. Each of the six subscales within each scale was summated and then divided by the total number of items in each to produce a mean score, interpreted from a low level of agreement on that particular subscale (1.0-3.0) to a high level of agreement $(>3.0)$.

\section{Analysis}

Data analyses were conducted using IBM SPSS V. 24.0 (2015). Descriptive statistics were used to analyze demographics of nurses employed in PC, characteristics of their places of employment and practice responsibilities. Chi-square analyses and $z$-test for proportions were used to determine between-group differences for categorical variables and to follow up significant results, respectively. Tables $1-3$ and Appendix 1 present comparisons by group (PC-Only vs. PC-Plus), and Appendix 2 displays proportion testing for pairwise comparisons by nurse type (all these elements are available online at longwoods.com/content/26130). For between-group comparisons of continuous variables, multivariate analyses of variance using Wilk's criterion $(\lambda)$ was the test statistic for the PHCE, JDIN and JDRN total summated scales and subscales. Significance was set at $p<0.05$ for all tests.

\section{Results}

Responses to questions about place of employment and practice area helped divide the nurse respondents into five distinct groups (Figure 1). Group A, "PC-Only" ( $n=192)$, identified 
their place of employment as PC and their area of practice as PC only. Group B, "PC-Plus" $(n=111)$, identified their place of employment as PC and their area of practice as PC plus at least one other area, most commonly community health and or acute care. Demographic information about the nurses in Groups A and B is provided in Table 1. Group C $(n=98)$ identified their place of employment as other than PC and area of practice as PC plus at least one other area, most commonly acute care, long-term care and hospice/end-of-life/palliative care. This group included five NPs (5.1\%), 42 RNs (42.9\%) and 52 LPNs (52.0\%). Group D identified their place of employment as other than PC, most commonly hospital, and their area of practice as PC-Only $(n=32)$. This group included six NPs $(18.8 \%), 15$ RNs $(45.9 \%)$ and 11 LPNs (34.4\%). Group E identified their place of employment as PC and their area of practice as other than PC $(n=293)$, most commonly community health $(169 ; 57.7 \%)$ and home care $(66 ; 22.5 \%)$. This group included $15 \mathrm{NPs}(5.1 \%), 240 \mathrm{RNs}(81.9 \%)$ and 38 LPNs (13\%). In consultation with our advisory group, we prioritized comparative analysis of the groups that most aligned with CIHI's definition of $\mathrm{PC}$ practice, namely, Groups A and B (PC-Only and PC-Plus). Data are available for the other groups from the corresponding author.

FIGURE 1. Overlapping definitions of a primary care nurse based on area(s) of current practice and primary place of employment

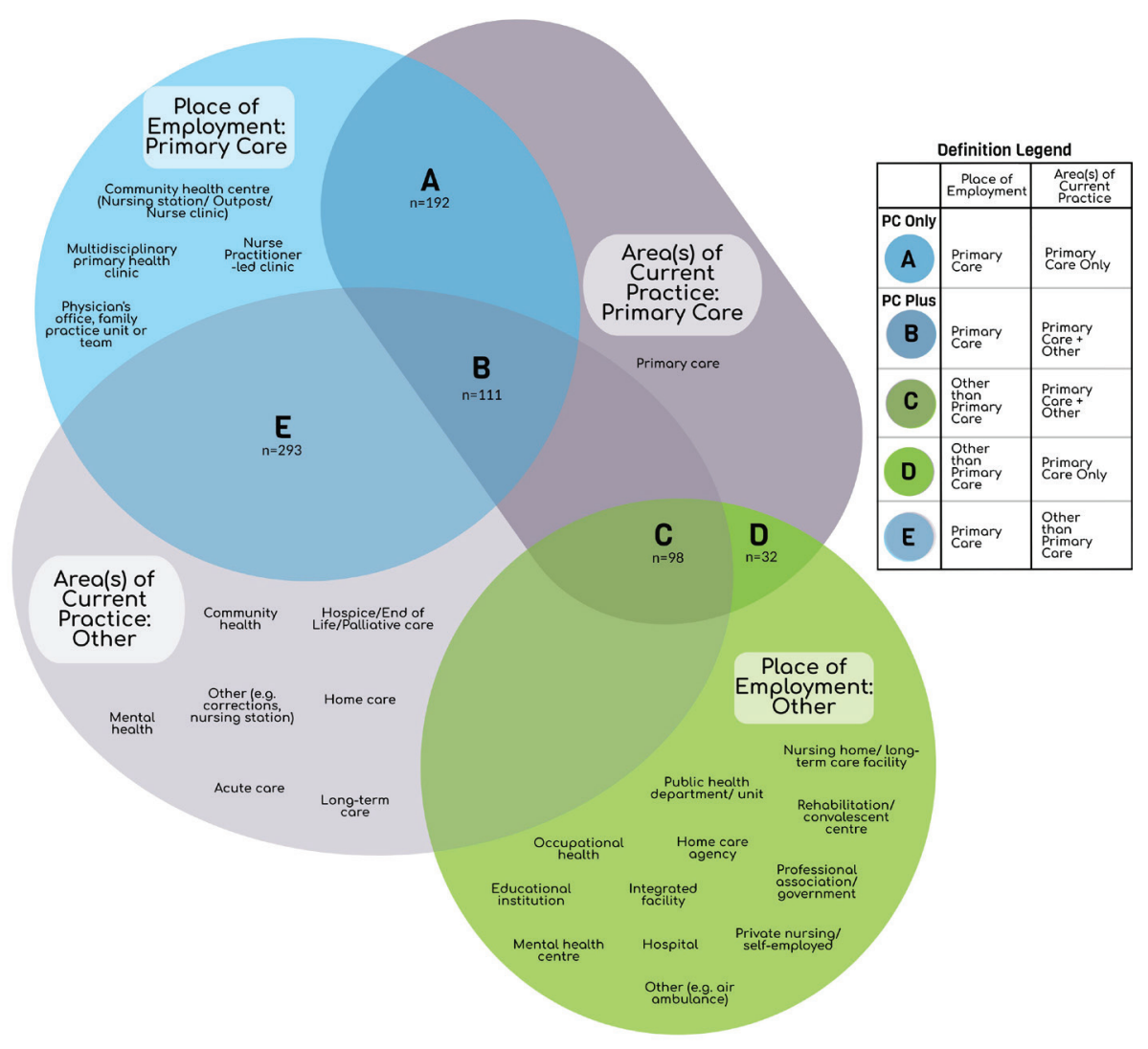


As Table 1 shows (available online at longwoods.com/content/26130), Group A had significantly higher proportions of NPs and LPNs and fewer RNs than Group B. Group A also had a higher proportion of nurses with graduate education and a lower proportion of nurses in the 30-34 and >60 age categories. Proportionally, there were more Ontario and Atlantic nurses in Group A and more Territories nurses in Group B.

As shown in Table 2 (available online at longwoods.com/content/26130), proportionally fewer nurses in Group A than in Group B worked in communities with a population of $<1,000$ and at a distance of $\geq 1,000 \mathrm{~km}$ to an advanced referral centre. Fewer PC-Only than PC-Plus nurses were required to be on call. In both Tables 1 and 2, Group A signifies PC-Only nurses and Group B is PC-Plus.

Table 3 (available online at longwoods.com/content/26130) shows that in the analysis of the PHCE, JDIN and JRIN summated scales, the combined PHCE dependent variable had a significant main effect for group $F(8,231)=8.55, p<0.001$, and partial $\eta^{2}=0.229$. Compared with nurses in Group A (PC-Only), nurses in Group B (PC-Plus) reported significantly higher scores on the summated PHCE scale and on the Accessibility/Availability and Population-Orientation subscales. Nurses in Group A (PC-Only) had significantly higher scores on the Quality Improvement subscale than nurses in Group B (PC-Plus).

The results from the full JRIN scale indicated there were statistically significant differences between groups $F(6,261)=4.09, p=0.001$, and partial $\eta^{2}=0.086$. Nurses in Group A (PC-Only) reported significantly higher scores on the summated JRIN scale and on the subscales of Supervision, Recognition and Feedback; Staffing and Time; Technology; and Training, Professional Development, and Continuing Education than nurses in Group B (PC-Plus). The level of perceived job-related resources for both Groups A and B was in the medium range.

The results from the full JDIN scale indicated there were statistically significant differences between groups $F(7,219)=2.68, p=0.011$, and partial $\eta^{2}=0.079$. Nurses in Group B (PC-Plus) reported significantly higher scores on the summated JDIN scale, the Equipment and Supplies subscale and the Safety subscale. The level of perceived demands related to their work for both groups was in the low-to-medium range.

Appendix 1 compares practice responsibilities of PC-Only and PC-Plus nurses, and is available at longwoods.com/content/26130. Proportionally fewer PC-Only nurses than PC-Plus nurses completed history-taking and physical examinations, independently made a nursing diagnosis, followed protocols/decision-support tools, dispensed medications and provided emergency care services and transportation. Although proportionally more PC-Only than PC-Plus nurses ordered advanced diagnostic tests and imaging, fewer performed and interpreted laboratory tests and diagnostic imaging on site. Box 1 outlines a fictional example of a PC-Plus nurse developed from survey results to illustrate this lesser-known practice group. 
BOX 1. Fictional example of a PC-Plus nurse developed from survey results to illustrate this lesserknown practice group

Susan, a Registered Nurse, works in a health centre in a small town. It is a multidisciplinary clinic with PC provider offices on one "side" of the building, a separate entrance for patients seeking public health services, and an urgent care entrance that is staffed until 5 p.m. Susan works in the urgent care area. While other PC providers see booked patients most of the day and one is "on call" for the urgent care side, Susan triages all patients who enter the clinic seeking care. Some patients she can manage independently and others she consults with or refers to team members. There are limited lab and X-ray services in the clinic, which Susan often must perform, and she maintains her skills in emergency nursing. The health centre is just off a major highway, so in addition to myocardial infarctions or serious occupational/ agricultural injuries coming through the door, Susan must be prepared to receive trauma victims from motor vehicle accidents or other similar events. Four mornings per month, she visits the assisted living complex across the road with another PC provider to help the staff with medication reconciliation and to complete assessments for residents that have new or chronic health concerns.

Recognizing that there are legislated scopes of practice, to explore what actually happens in practice, albeit through self-report data, we analyzed the sample by nurse type, (i.e., NP, RN and LPN). This full analysis is available in Appendix 2 (available at longwoods.com/ content/26130). Proportionally more NPs than RNs completed history-taking and physical examinations and independently made a medical diagnosis; prescribed medications; ordered, performed, interpreted and followed up advanced diagnostic tests; ordered, interpreted and followed up diagnostic imaging; and made referrals to medical specialists. Proportionately fewer NPs than RNs dispensed medications, prescribed using protocols, performed laboratory tests and diagnostic imaging, and were involved in emergency care and transportation. Comparing RNs and LPNs, proportionately more RNs performed all practice responsibilities except ordering advanced diagnostic tests and advanced imaging, which few nurses of either type did.

\section{Discussion}

This study provides new information about nurses providing PC in rural and remote Canada, the context of their practice and their practice responsibilities. The distances involved and the small number of nurses offering PC to large geographically dispersed populations are staggering. Our results highlight the vital role nurses have in not only the delivery of a broad range of PC services but also their engagement and partnership with communities to improve health and health determinants. Our results illuminate the complex nature of $\mathrm{PC}$ in rural and remote Canada as well as the gaps in resources and services that should be enhanced to provide PC.

Nurses' responses to survey questions about their place of employment and practice focus reveal the diverse nature of their roles in PC and the important influence of context on practice (Hogg et al. 2008). Although nurses are regarded as the backbone of rural and remote healthcare, there is surprisingly little research about their practice in PC (MartinMisener et al. 2014). This may explain the lack of focused attention to the nursing workforce in policy discussions about rural and remote PC. Filling this information gap is critical for decision-makers responsible for healthcare planning and delivery. 
Our results reveal clear differences between nurses in $\mathrm{PC}$ workplaces who report $\mathrm{PC}$ as their only practice focus (PC-Only) and nurses who report $\mathrm{PC}$ as one of additional practice foci (PC-Plus). The features of the workplaces of PC-Only (Group A) nurses (i.e., more NPs, graduate education and communities with 5,000 plus people and within $500 \mathrm{~km}$ of a major referral centre) resemble interprofessional team-based models of $\mathrm{PC}$ also seen in urban areas (Hutchison et al. 2011). In contrast, PC-Plus nurses' (Group B's) workplace features (i.e., more RNs, baccalaureate education communities with $<1,000$ people and distance $>1,000 \mathrm{~km}$ from major referral centres) are suggestive of health centres in more remote areas (Martin-Misener et al. 2008).

Nurses in both groups perceived engagement of their workplace in PHC delivery to be high; however, the PC-Plus nurses' scores were higher overall, and for Accessibility and Population-Orientation subscales (Kosteniuk et al. 2017). Population orientation refers to "the extent to which primary care providers assess and respond to the health needs of the population they serve" (Haggerty et al. 2007, p. 340). PC-Plus nurses were more likely than PC-Only nurses to report a good fit between their workplace and community needs. One interpretation is that because many PC-Plus nurses worked in remote communities in which multiple services are offered in one location (e.g., PC, public health and emergency care), they could perceive their workplace to be more oriented and responsive to population needs. PC-Only nurses had higher scores on the Quality Improvement subscale. This could be related to this group having a higher proportion of nurses with graduate education and/or having available staff, time and technology resources. Another possibility is that the more traditional workplaces of PC-Only nurses may have been influenced by the increasing focus on healthcare improvement in PC promoted by organizations such as the Canadian Foundation for Health Care Improvement and Accreditation Canada.

Nurses in Group B reported higher job-related demands and had lower perceived jobrelated resources than PC-Only nurses. Demands were particularly high with respect to safety (e.g., their personal safety being at risk) and equipment and supplies (e.g,, necessary equipment or supplies being unavailable or in poor condition), issues that have been reported in previous studies (MacLeod et al. 2008; Martin-Misener et al. 2008). Interestingly, demands such as comfort with working conditions (which included items related to workload), preparedness/scope of practice and isolation were not significantly different between groups, even though higher proportions of PC-Plus nurses had on-call responsibilities and provided emergency care and transportation. It is possible that the differences in JRIN and JDIN results are related to the population size and geographic location of the communities in which nurses worked. Relative to each other, more PC-Plus than PC-Only nurses were employed in smaller-sized and more remote communities.

In our study, NPs' practice responsibilities aligned with their legislated scope of practice (CCRNR 2015). Not unexpectedly, some RNs had responsibilities extending beyond their legislated scope of practice. PC-Plus nurses, most of whom were baccalaureate-educated RNs, were employed in some of the smallest and remotest communities with high overall 
job-related demands and fewer resources. That proportionally more PC-Plus than PC-Only nurses dispensed medications, performed and analyzed laboratory tests on-site, and provided emergency care and transportation is consistent with the role descriptions of nurses employed in communities without other on-site personnel to offer these services (MartinMisener et al. 2008). It is possible that PC-Plus nurses who were the group more likely to be the recipients of task-shifting had different JRIN and JDIN scores because of this. The roles and responsibilities of these RNs encompass task shifting from not only physicians and NPs but also pharmacists, laboratory technicians and paramedics. Notwithstanding the small number of LPN participants, this study identifies previously undocumented task-shifting from RNs to LPNs in PC that requires monitoring.

Resourcing PC is a pressing equity concern given the overlap between rural and remote residence and Indigenous populations (Marchildon 2018). Task-shifting has long been a necessary strategy to improve access to rural and remote PC and may become even more common with movement toward team-based care in PC. In rural and remote communities, where team composition and size differ from urban areas, the work of nurses in filling gaps in access to other team members cannot remain invisible. Rather, the associated changes in nurses' roles and responsibilities must be accompanied by education and regulation to safeguard patient safety and quality of care (Freund et al. 2015; Moffit et al. 2018). Consideration of the skill mix of other team members is also needed to promote high-quality $\mathrm{PC}$ in rural and remote communities. Attending to these supports and increasing the number of NPs are approaches to prevent, or at least reduce, a double standard of care between rural/remote and urban communities (Tarlier and Browne 2011).

Lastly, our study has implications for optimizing measurement of PC performance in rural and remote Canada. The variability in how nurses responded to questions about primary place of employment and area(s) of practice turned what initially seemed like a straightforward approach to determine the subsample of interest into unanticipated complexity. Important first steps are to consider how to expand CIHI's 2012 definition of PC to be more inclusive of the workplaces where rural and remote PC nurses are employed and for nurse regulators to develop consistency in defining PC in registration databases. More information is needed about the other three groups of nurses identified in our study (Figure 1). The largest of these groups was nurses who identified that their place of employment was PC but that their area of practice was other than PC $(n=293)$. This could potentially represent nurses who are employed in PC settings, but have a different practice focus, for example, mental health or public health. Qualitative research to examine nurses' understanding of the nature of $\mathrm{PC}$ would be useful to inform definition changes.

This study was pan-Canadian in scope. A limitation is the small number of respondents from Quebec despite the survey being available in French. The inclusion of nurses in Whitehorse, Iqaluit and Yellowknife, despite the small number, may have diluted differences between Groups B and A. Because we did not define any area of practice, including PC, some nurses may have been unclear about what PC is. This may have influenced their responses to the survey, resulting in under- or overreporting of employment in PC. 


\section{Conclusion}

The contributions of the rural and remote nursing workforce to PC include a mosaic rendered invisible by contemporary characterizations and interpretations. Current approaches to defining PC workplaces are insufficient to identify nurses providing PC in rural and remote Canada, limiting evaluation and improvement initiatives.

\section{Acknowledgement}

This article stems from the study Nursing Practice in Rural and Remote Canada II, led by M. MacLeod, N. Stewart and J. Kulig (http://ruralnursing.unbc.ca). We acknowledge the funding from the Canadian Institutes of Health Research and the other in-kind funding. We thank the Advisory Team led by Penny Anguish of Northern Health, and the nurses who responded to the survey. We also thank Leana Garraway, Steinunn Jónatansdóttir and Alexandra Thomlinson for their contributions.

Correspondence may be directed to: Ruth Martin-Misener. Her e-mail address is ruth.martinmisener@dal.ca.

\section{References}

Barnes, H., M.R. Richards, M.D. McHugh and G. Martsolf. 2018. Rural and Nonrural Primary Care Physician Practices Increasingly Rely on Nurse Practitioners. Health Affairs (Millwood) 37(6): 908-914. doi:10.1377/hlthaff.2017.1158.

British Columbia Ministry of Health. 2015. Rural Health Services in BC: A Policy Framework to Provide a System of Quality Care. Retrieved January 30, 2019. <https://www.health.gov.bc.ca/library/publications/ year/2015/rural-health-policy-paper.pdf>.

Canadian Council of Registered Nurse Regulators (CCRNR). 2015. Nurse Practitioner Practice Analysis. Retrieved November 30 2018. <http://www.ccrnr.ca/nurse-practitioners.html>.

Canadian Institute for Health Information (CIHI). 2012. Pan-Canadian Primary Healthcare Indicator Report Update. Ottawa, ON. Retrieved January 30, 2019. <www.cihi.ca>.

Canadian Institute for Health Information (CIHI). 2019a. Regulated Nurses 2018 Canada and Jurisdictional Highlights. Retrieved October 30, 2019. <www.cihi.ca>.

Canadian Institute for Health Information (CIHI). 2019b. Regulated Nurses 2018 Methodology Guide. Retrieved October 30, 2019. <www.cihi.ca>.

Canadian Nurses Association (CNA). 2017. Nurse Practitioners. Retrieved January 30, 2019. <https://www. cna-aiic,ca/en/nursing-practice/the-practice-of-nursing/advanced-nursing-practice/nurse-practitioners $>$.

du Plessis, V., R. Beshiri, R.D. Bollman and H. Clemenson. 2001. Rural and Small Town Canada Analysis Bulletin, Definitions of Rural. Retrieved January 30, 2019. <https://www150.statcan.gc.ca/n1/en/pub/21-006$\mathrm{x} / 21-006-\mathrm{x} 2001003$-eng.pdf?st=BNNRTf-P $>$.

Freund, T., C. Everett, P. Griffiths, C. Hudon, L. Naccarella and M. Laurant. 2015. Skill Mix, Roles and Remuneration in the Primary Care Workforce: Who Are the Healthcare Professionals in the Primary Care Teams across the World? International Journal of Nursing Studies 52(3): 727-43. doi:10.1016/j. ijnurstu.2014.11.014.

Haggerty, J., F. Burge, D. Gass, J.F. Lévesque, M.D. Beaulieu, R. Pineault et al. 2007. Operational Definitions of Attributes of Primary Health Care: Consensus among Canadian Experts. Annals of Family Medicine 5: 336-44. doi:10.1370/afm.682. 
Hogg, W., M. Rowan, G. Russell, R. Geneau and L. Muldoon. 2008. Framework for Primary Care

Organizations: The Importance of a Structural Domain. International Journal for Quality in Health Care 20(5): 308-13. doi:10.1093/intqhe/mzm054.

Hutchison, B., J.F. Levesque, E. Strumpf and N. Coyle. 2011. Primary Health Care in Canada: Systems in Motion. Millbank Quarterly 89(2): 256-88. doi:10.1111/j.1468-0009.2011.00628.x.

Hutchison, B. 2013. Reforming Canadian Primary Care - Don't Stop Half-Way. Healthcare Policy 9(1): 12-25. doi:10.12927/hcpol.2013.23481.

IBM Corp. Released 2016. IBM SPSS Statistics for Windows, Version 24.0. Armonk, NY: IBM Corp.

Kosteniuk, J.G., N.J. Stewart, C.P. Karunanayake, E.C. Wilson, K.L. Penz, J.C. Kulig et al. 2017. Exploratory Factor Analysis and Reliability of the Primary Health Care Engagement (PHCE) Scale in Rural and Remote Nurses: Findings from a National Survey. Primary Health Care Research and Development 18(6): 608-22. doi:10.1017/S146342361700038X.

Kulig, J.C., M.E. Andrews, N.L. Stewart, R. Pitblado, M.L.P. MacLeod and D. Bentham et al. 2008. How do Registered Nurses Define Rurality? Australian Journal of Rural Health 16: 28-32.

doi: 10.1111/j.1440-1584.2007.00947.

MacLeod, M., J. Kulig, N. Stewart and R. Pitblado. 2004. Rural and Remote Nursing Practice: Final Report to Canadian Health Services Research Foundation. Retrieved February 7, 2020. <https://www.unbc.ca/sites/ default/files/sections/rural-nursing/en/17-rrnfinalreport.pdf>.

MacLeod, M., R. Martin-Misener, K. Banks, M.A. Morton, C. Vogt and D. Bentham. 2008. I'm a Different Kind of Nurse: Advice from Nurses in Rural and Remote Canada. Canadian Journal of Nursing Leadership 21(3): 40-53. doi:10.12927/cjnl.2008.20060.

MacLeod, M., J. Kulig, N. Stewart, P. Anguish, M.E. Andrews, D. Banner et al. 2017. Who Are the Nurses that Work in Rural and Remote Communities in Canada and Why Do They Stay? Results of a National Survey. Human Resources for Health 15(1): 34. doi:10.1186/s12960-017-0209-0.

MacLeod, M.L.P., N.J. Stewart, J.G. Kosteniuk, K.L. Penz, J. Olynick, C.P. Karunanayake et al. 2019. Rural and Remote Registered Nurses' Perceptions of Working beyond Their Legislated Scope of Practice. Nursing Leadership 32(1): 8-19. doi:10.12927/cjnl.2019.25852.

Maier, C.B. and L.H. Aiken. 2016. Task Shifting from Physicians to Nurses in Primary Care in 39 Countries: A Cross-Country Comparative Study. The European Journal of Public Health 26(6): 927-34. doi:10.1093/ eurpub/ckw098.

Marchildon, G.P. 2018. A Policy Research Agenda for Health Systems in Canada's North. Healthcare Papers 17(3): 35-40. doi:10.12927/hcpap.2018.25503.

Martin, D., A.P. Miller, A. Quesnel-Vallée, N.R. Caron, B. Vissandjée and G.P. Marchildon. 2018. Canada's Universal Health-Care System: Achieving its Potential. The Lancet 391(10131): 1718-35. doi:10.1016/ S0140-6736(18)30181-8.

Martin-Misener, R., M. MacLeod, K. Banks, A. Michel Morton, C. Vogt and D. Bentham. 2008. There's Rural, and Then There's Rural: Advice from Nurses Providing Primary Healthcare in Northern Remote Communities. Canadian Journal of Nursing Leadership 21(3): 40-53. doi:10.12927/cjnl.2008.20062.

Martin-Misener, R., D. Bryant-Lukosius, C. Bullard, D. Campbell, N. Carter, F. Donald et al. 2014. Optimizing the Role of Registered Nurses and Nurse Practitioners in Primary Care: Final Report. Canadian Nurses Association. Retrieved January 30, 2019. <http://www.cna-aiic.ca/en/professional-development/canadian-networkof-nursing-specialties/whats-new-with-the-network/network-news-room/news-about-the-network/2014/ optimizing-rn-roles>.

Moffit, P., R. Munday and J. Inman. 2018. Professionalism and Professional Conduct in the Northwest Territories and Nunavut Canada. In H. Exner-Pirot, B. Norbve and L. Butler (Ed.), Northern and Indigenous Health and Healthcare (pp. 249-59). Saskatoon, Saskatchewan: University of Saskatchewan.

Nurses and Nurse Practitioners of British Columbia (NNPBC). 2018. Enhancing Rural and Remote Nursing Practice for a Healthier B.C. Retrieved January 30, 2019. <https://www.nnpbc.com/pdfs/policy-and-advocacy/ rural-and-remote/Enhancing-Rural-\&-Remote-Nursing-Practice-for-a-Healthier-BC.pdf >. 
Penz, K.L., J.G. Kosteniuk, N.L. Stewart, M.L.P. MacLeod, J.C. Kulig, C.P. Karunanayake et al. 2018.

Development and Psychometric Evaluation of the Job Demands in Nursing Scale and Job Resources in Nursing Scale: Results from a National Study. Nursing Open 6(2): 348-66. doi:10.1002/nop2.215.

Smith, J.C. and V. Vandall-Walker. 2017. A Double Whammy! New Baccalaureate Registered Nurses' Transitions into Rural Acute Care. Rural and Remote Health 17(4): 4256. doi:10.22605/RRH4256.

Starfield, B. 1992. Primary Care: Concept, Evaluation, and Policy. New York, NY: Oxford University Press.

Statistics Canada. 2017. Primary Health Care Providers, 2016. Ottawa, ON. Retrieved January 30, 2019. $<$ https://www150.statcan.gc.ca/n1/pub/82-625-x/2017001/article/54863-eng.htm>.

Stewart, N.J., C. D’Arcy, J.R. Pitblado, D.G. Morgan, D. Forbes, G. Remus et al. 2005. A Profile of Registered Nurses in Rural and Remote Canada. Canadian Journal of Nursing Research 37(1). Retrieved February 7, 2020. $<$ https://cjnr.archive.mcgill.ca/article/view/1930>.

Tam, T. 2017. The Chief Public Health Officer's Report on the State of Public Health in Canada 2017 Designing Healthy Living. Retrieved February, 2020. <https://www.canada.ca/en/public-health/services/ publications/chief-public-health-officer-reports-state-public-health-canada/2017-designing-healthy-living.html>. Tarlier, D. and A. Browne. 2011. Remote Nursing Certified Practice: Viewing Nursing and Nurse Practitioner Practice through a Social Justice Lens. Canadian Journal of Nursing Research 43(2): 38-61.

Tomblin Murphy, G., A. MacKenzie, R. Alder, S. Birch, G. Kephart and L. O’Brien-Pallas. 2009. An Applied Simulation Model for Estimating the Supply of and Requirements for Registered Nurses Based on Population Health Needs. Policy Politics Nursing Practice 10(4): 240-51. doi:10.1177/1527154409358777.

Xue, Y., J. A. Smith and J. Spetz. 2019. Primary Care Nurse Practitioners and Physicians in Low-Income and Rural Areas, 2010-2016. JAMA 321 (1): 102-05. doi:10.1001/jama.2018.17944.

\section{Join the conversation}

\section{@longwoodsnotes}

\section{YouTube youtube.com/LongwoodsTV}

(2) pinterest.com/longwoods

facebook.com/LongwoodsPublishingCorporation

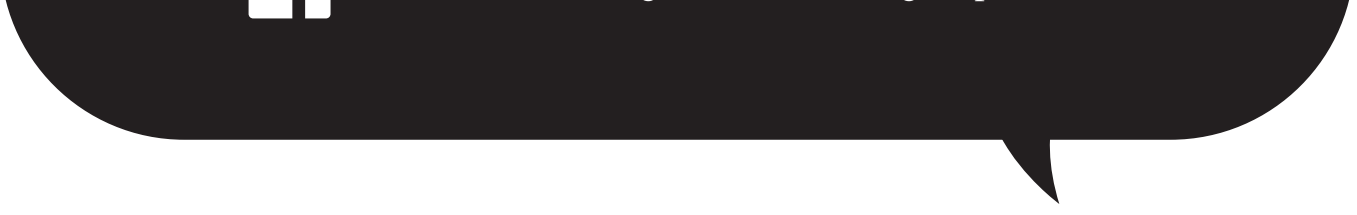

